\title{
Temporal Trend of Urolithiasis Incidence in China: An Age-Period-Cohort Analysis
}

\author{
Hui Huang' \\ Minmin $\mathrm{Li}^{2}$ \\ Hourui Fan (iD ${ }^{3}$ \\ Ruhai Bai id 4 \\ 'Department of Dermatology, The First \\ Affiliated Hospital, Jinan University, \\ Guangzhou, Guangdong, People's \\ Republic of China; ${ }^{2}$ Department of \\ Nutrition and Food Hygiene, Shaanxi \\ Provincial Center for Disease Control \\ and Prevention, Xi'an, Shaanxi, People's \\ Republic of China; ${ }^{3}$ Health Science \\ Center, Xi'an Jiaotong University, Xi'an, \\ Shaanxi, People's Republic of China; \\ ${ }^{4}$ School of Public Affairs, Nanjing \\ University of Science and Technology, \\ Nanjing, Jiangsu, People's Republic of \\ China
}

Purpose: Urolithiasis is one of the commonest disease of the urinary system. This study aimed to assess the long-term urolithiasis incidence trends in China between 1990 and 2019. Patients and Methods: The incidence data of urolithiasis were extracted from Global Burden of Disease Study 2019, and an age-period-cohort framework was used to estimate the age, period, and cohort effects.

Results: We found that the net drift was $-2.72 \%$ (95\% CI: $-2.84 \%$ to $-2.60 \%)$ per year for men and $-2.14 \%(95 \% \mathrm{CI}:-2.24 \%$ to $-2.05 \%)$ per year for women, and except men in age group $0-4$, the local drift values were below 0 in all age groups $(\mathrm{P}<0.05$ for all $)$ in both sexes during the period of 1990 to 2019. In the same birth cohort, the risk of incidence from urolithiasis rose first and then decreased with age for both sexes after controlling for period deviations, and in addition, after 20 years old, the risk of incidence of urolithiasis in men was higher than that in women (significantly with $\mathrm{P}<0.05$ ). In general, the estimated period and cohort relative risks were found in similar downward patterns for both sexes.

Conclusion: In the past 30 years, the age-standard incidence of urolithiasis in both sex of Chinese people has decreased, but the crude incidence of urolithiasis in Chinese women has increased. Considering the aging of population structure in China, the overall number of female urolithiasis patients may increase, contributes to higher crude incidence rate. The problem of urolithiasis in Chinese women needs to be paid more attention.

Keywords: age-period-cohort analysis, urolithiasis, urinary stones, urolithiasis in China

\section{Introduction}

Urolithiasis refers to any stones in the urinary system including kidneys, ureters, bladder or urethra. It is one of the commonest diseases of the urinary system, usually accompanied by acute severe unilateral loin pain, nausea, hematuria and other symptoms, which will increase the urinary tract and the risk of infection complicated by uremic shock, acute kidney injury and even adverse renal outcome including end-stage renal disease (ESRD). ${ }^{1-3}$ The direct costs and indirect costs incurred by its treatment have brought heavy economic burdens to patients and society. ${ }^{4,5}$

Globally, due to climate warming, dietary changes, and obesity, the incidence and prevalence of urinary stones are rising around the world. ${ }^{5}$ As the main component of urolithiasis, China has witnessed a steady rise in the prevalence of kidney stones from 4\% to $6.4 \%$ from 2008 to $2014 .^{6-8}$ During May 2013 to July 2014, 1 out of 17 adults in China suffered from kidney stones. ${ }^{7}$ China has the largest population in the world; thus, the number of urolithiasis patients in China could be enormous. Assessing the nationwide epidemiology of urolithiasis is
Correspondence: Ruhai Bai

School of Public Affairs, Nanjing

University of Science and Technology, No.

200, Xiaolingwei Street, Nanjing, Jiangsu,

People's Republic of China

Email ruhaibai@hotmail.com 
important to assess the disease burden and developing relevant policies. However, there have been few studies of the trends in the incidence of urolithiasis in China. Furthermore, there has been no comprehensive analysis of the possible reasons underlying the temporal trends.

In this study, we used data from the Global Burden of Disease Study (GBD) 2019 and age-period-cohort framework to investigate the long-term trends in the incidence of urolithiasis in China, and examined the effects of age, period, and cohort from 1990 to 2019. Our research results may provide guidance for health resource allocation and policy formulation to strengthen prevention and treatment of high-risk populations.

\section{Materials and Methods}

\section{Data Source}

Data used in this study was extracted from GBD 2019, which has provided internally consistent estimates of age- and sexspecific all-cause and cause-specific incidence for 369 diseases for 204 countries and territories from 1990 to $2019 .{ }^{9}$ GBD 2019 synthesises a large and growing number of data input sources including surveys, censuses, vital statistics, and other health-related data sources. A Bayesian metaregression modelling tool, DisMod-MR 2.1, was used to ensure consistency of incidence for most causes. ${ }^{9}$

GBD 2019 data on China mainly come from two sources: surveillance data from the China Disease Surveillance Points system (DSPs) and Vital Registration (VR) data collected by the Chinese Center for Disease Control and Prevention. ${ }^{9}$ DSPs covers $24.3 \%$ of the country's total population since 2013, and VR accounting for roughly $8 \%$ of the national population. ${ }^{10}$ Because of the sampling strategy, the DSPs can represent the population of China. ${ }^{10,11}$ The VR system, while not representative, was able to give more accurate information due to specific causes and larger sample than the disease surveillance points. ${ }^{10}$ Together, the disease surveillance points and vital registration systems provided a nationally representative picture of health information in China. For GBD data, many steps, including under-reporting, misclassification, and incompleteness corrections, as well as the redistribution of the garbage codes to enhance the data quality, the bias reduced to some extent compared with that of research using raw data. ${ }^{12}$

Urolithiasis was diagnosed and defined based on the ninth and tenth revisions of the International Classification of Disease (ICD10: N20-N23.0; ICD9: 592-592.9, 594-594.9, 788.0). ${ }^{9}$ In this study, the incidence rate of urolithiasis were age-standardized by global age-standard population for both men and women. ${ }^{9}$

\section{Statistical Analysis}

Age-period-cohort (APC) frame work was used in this study to assess the age, period, and cohort effect on urolithiasis incidence rate, which can help analyze possible reasons underlying the temporal trends. The APC can be considered essentially as a multiple regression model which was developed based on the Poisson distribution. The specific equation was exhibited as follows: ${ }^{13}$

$\mathrm{Y}=\mu+\alpha \mathrm{A} \geq \mathrm{Y}_{\mathrm{i}}+\beta$ Period $_{\mathrm{j}}+\gamma$ Cohort $_{\mathrm{k}}+\varepsilon$

where $\mathrm{Y}$ denotes the response variable - the net effect on urolithiasis incidence for groups, $\alpha, \beta$, and $\gamma$ denoted the coefficient of age, period, and cohort of the APC model, respectively, and denoted the intercept of the model, and $\mu$ denoted the residual of the APC model.

The age effect represents the different risk in different age group. The period effect represents the risk difference in different periods. Cohort effect represents the risk difference among different birth cohort. By using APC model, the following parameters were estimated. Net drift, indicates the overall annual percentage change in study period. Local drifts, indicate annual percentage changes for each age group in study period. Longitudinal age curve indicates the period deviations adjusted age-specific rates in the reference cohort. The period (or cohort) relative risk (RR) indicates the period RR (or cohort RR) adjusted for age and nonlinear cohort (or period) effects compared with the reference period (or cohort). ${ }^{12}$

To conduct APC analysis, the incidence and population data were arranged into consecutive 5 years period from 1990 to 1994 (median 1992) to 2015-2019 (median 2017), and successive 5 year age interval from 0 to 4 years to 85 to 89 years. An R program provided by USA National Cancer Institute was used to estimate the parameters. ${ }^{14}$ The central age group, period, and birth cohort were defined as the reference in all analyses. ${ }^{15}$ Estimable functions were assessed by Wald $\chi 2$ tests. All statistical tests were 2-tailed and $\mathrm{P}<0.05$ were regarded as significant.

\section{Results}

Trends of the Incidence Rates for Urolithiasis by Sex for the Period of 1990 to 2019

From 1990 to 2019, the crude incidence rate (CIR) and age-standardized incidence rate (ASIR) of urolithiasis by 


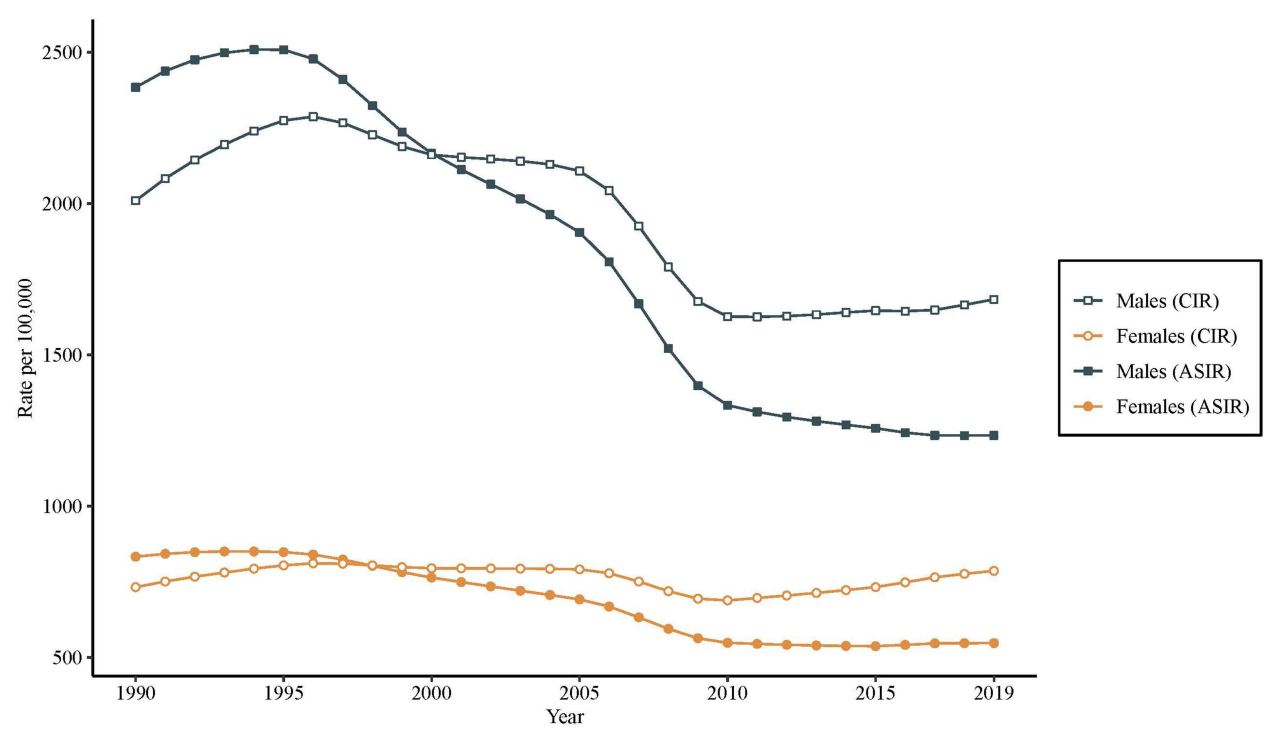

Figure I Trends of the age-standardized incidence rates (ASIR) and the crude incidence rates (CIR) per 100,000 populations for urolithiasis by sex in China, 1990 to 2019. Using the GBD 2019 (Global Burden of Disease Study 2019) global age-standard population.

gender were shown in Figure 1 and Supplementary Table S1. In general, the CIR of urolithiasis in male had shown a decline, from 2009.227 per 100,000 in 1990 to 1683.201 per 100,000 in 2019. The CIR in women rose from 732.421 per 100,000 in 1990 to 786.3067 in 2019. The ASIR in male urolithiasis showed a decline, from $2383.901 / 100,000$ in 1990 to $1233.800 / 100,000$ in 2019 , and the ASIR in female urolithiasis decreased from $833.128 / 100,000$ in 1990 to $547.489 / 100,000$ in 2019 . In addition, the sex differences in the incidence of urolithiasis have decreased over the past 30 years.

\section{Local Drift with Net Drift Values for Urolithiasis Incidence in China}

The overall annual change percentage (net drif) of the incidence of urolithiasis and the annual change of the incidence of urolithiasis in each age group (local drift) were shown in Figure 2. For the overall annual change percentage of the incidence of urolithiasis, net drift for men was $-2.72 \%(95 \%$ CI: $-2.84 \%,-2.60 \%$ ), and net drift for women was $-2.14 \%$ (95\% CI: $-2.24 \%,-2.05 \%)$. Regarding the annual change of each age group, the local drift of women in each age group was lower than 0 (significantly with $\mathrm{P}<0.05$ ), and the lowest local drift value was found in the age group 0 to 4 years, which was $-3.26 \%$ (95\% CI: $-4.63 \%,-1.87 \%)$. Except for men aged 0 to 4 , the local drift of all other age groups was lower than 0 (significantly with $\mathrm{P}<0.05$ ). Among them, local drift value was lowest in the age group 65 to 69 years, with $-4.43 \%$ (95\% CI: $-4.56 \%,-4.30 \%)$.

\section{The Longitudinal Age Curves of Urolithiasis Incidence by Sex in China}

Figure 3 and Supplementary Table S2 showed the longitudinal age curve of the risk of urolithiasis. In the same birth cohort, the risk of urolithiasis in female showing a reversed V-shape with increase of age. The highest risk of urolithiasis in women was found in age group 50 to 54 years, with 1661.7/100,000 (95\% CI: 1631.0, 1693.1). Similar to women, the risk of urolithiasis in men also showing a reversed V-shape. The highest risk of urolithiasis in men was found in age group 50 to 54, with 3568.9 per 100,000 (95\% CI: $3488.0,3651.6)$. In addition, after the age of 20 , the risk of urolithiasis in men was higher than that in women (significantly with $\mathrm{P}<0.05$ ).

\section{Period and Cohort Relative Risks of Urolithiasis Incidence Rate by Sex in China}

Figures 4 and 5 showed the estimated period RRs and cohort RRs by gender. The effect of period effect on the incidence of urolithiasis showed a similar trend between men and women (Figure 4 and Supplementary Table S3). Compared with the reference period (year 2000 to 2004), the period RRs for both men and women had decreased after 2005. The cohort RRs showed a downward pattern for both males and females from earlier to later birth cohort overall (Figure 5 and Supplementary Table S4). 


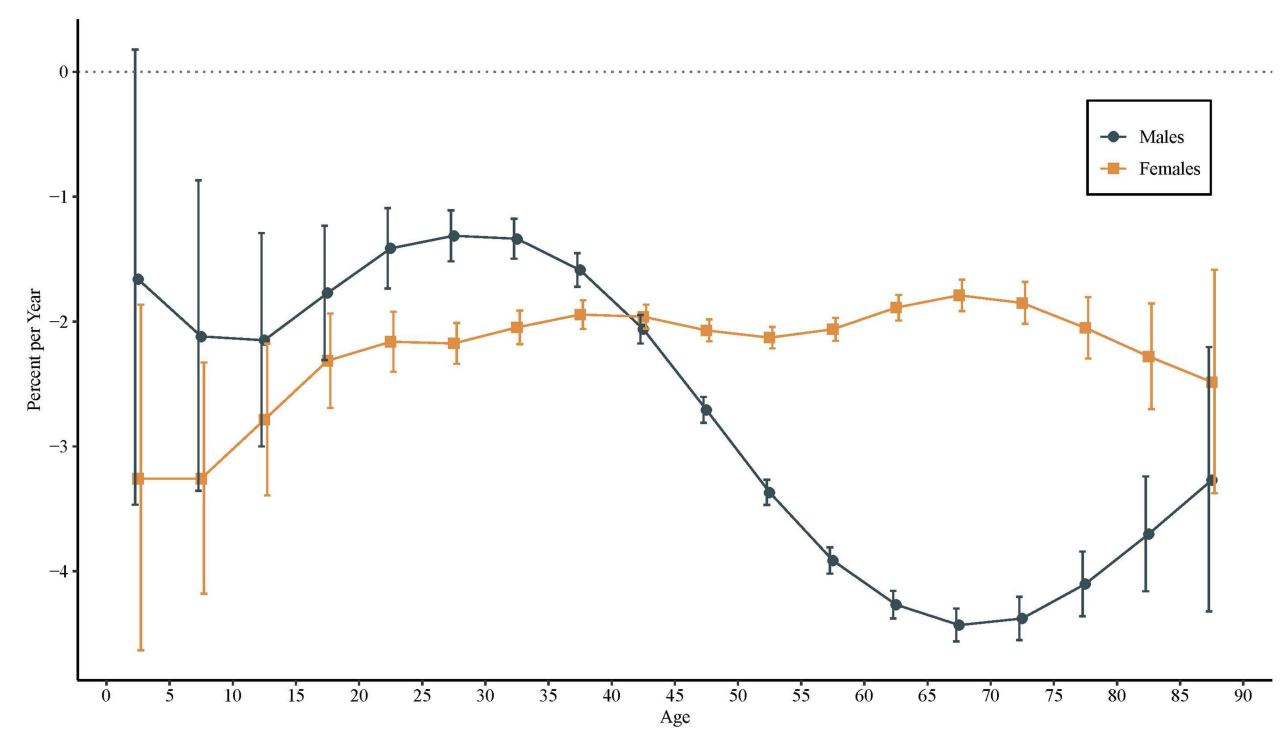

Figure $\mathbf{2}$ Local drift with net drift values for urolithiasis incidence in China. Age group-specific annual percent change (local drift) with the overall annual percent change (net drift) in urolithiasis incidence rate and the corresponding $95 \%$ confidence intervals.

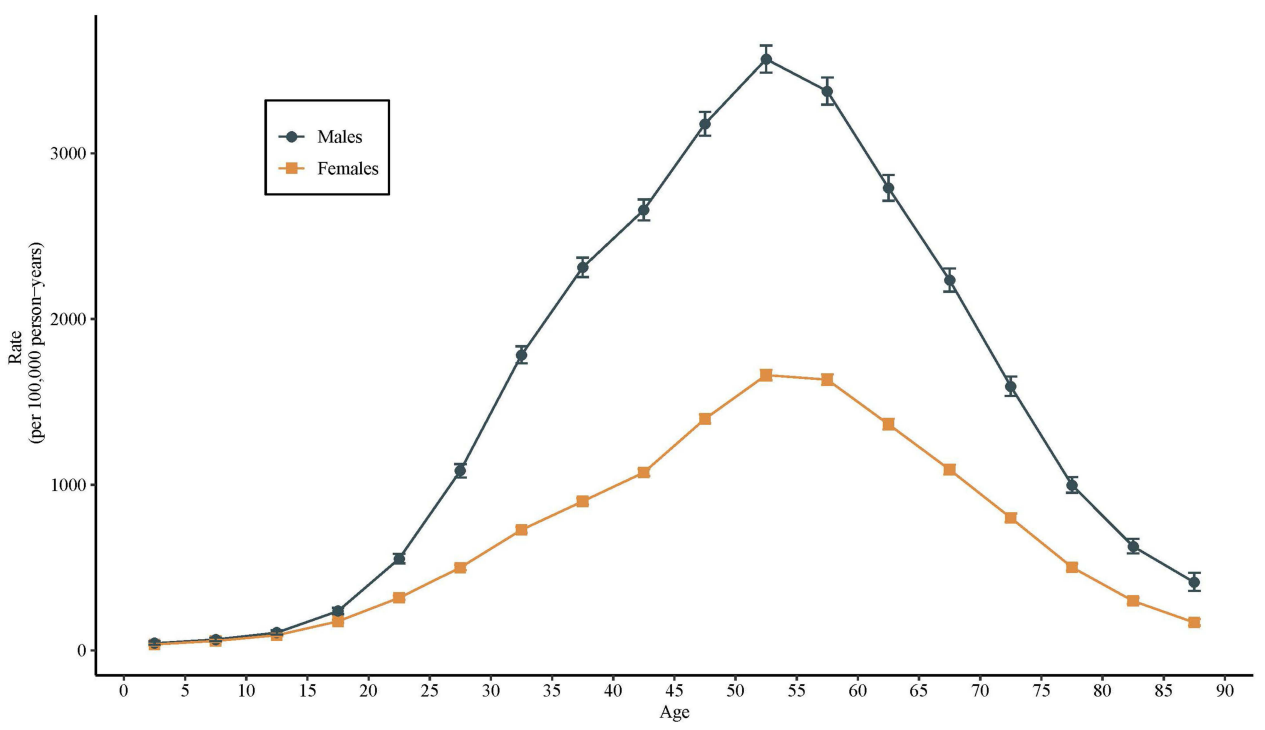

Figure 3 Longitudinal age curves of urolithiasis incidence in China. Fitted longitudinal age-specific rates of urolithiasis incidence (per 100,000 person-years) and the corresponding $95 \%$ confidence intervals (some of them were too narrow to show in the figure).

\section{Discussion}

As far as we know, there were few researches to analyze the incidence trend of urolithiasis in China. This study was the first study to explore the incidence trend of urolithiasis in China from 1990 to 2019 using the APC framework. The results of this study showed that, the CIR of urolithiasis in Chinese men had generally shown a decline from 1990 to 2019, while the CIR of urolithiasis in Chinese women had shown an increase during the same period. The ASIR of urolithiasis in both Chinese men and women had shown a decline. For each age group, except age group 0 to 4 , the incidence of urolithiasis in all age groups showed a decreasing pattern in both men and women.

Our study found that the ASIR of urolithiasis had shown a decline in both Chinese men and women, this result is consistent with previous studies. ${ }^{16,17}$ The reduction in the ASIR of urolithiasis in China may related to the changes in China's diet structure in recent years. In the past 20 years, the consumption of fruits and vegetables by Chinese children and adults had shown an increase. ${ }^{18,19}$ A diet rich in fruits and vegetables could significantly reduce the risk of kidney stones. ${ }^{20}$ Fresh vegetables are rich in fiber, among which phytic 


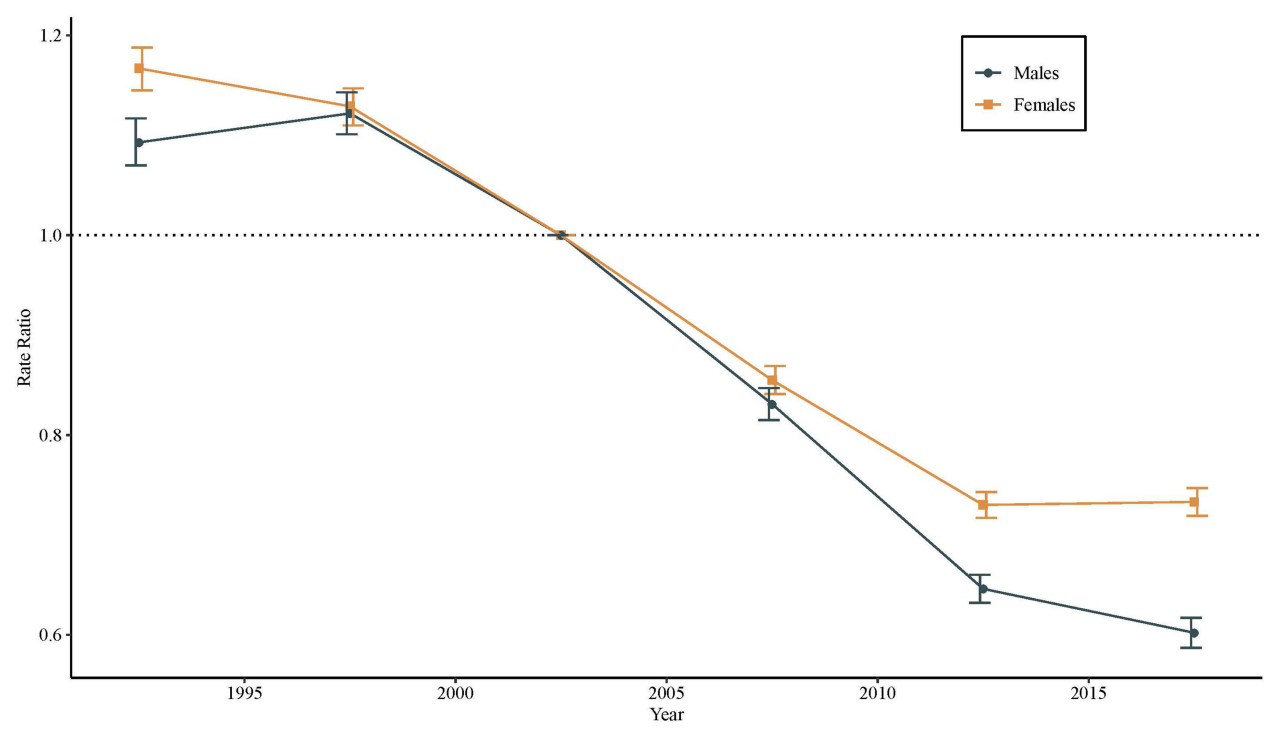

Figure 4 Period relative risks (RRs) of urolithiasis incidence rate by sex in China. The relative risk of each period compared with the reference one (year 2000 to 2004) adjusted for age and nonlinear cohort effects and the corresponding $95 \%$ confidence intervals.

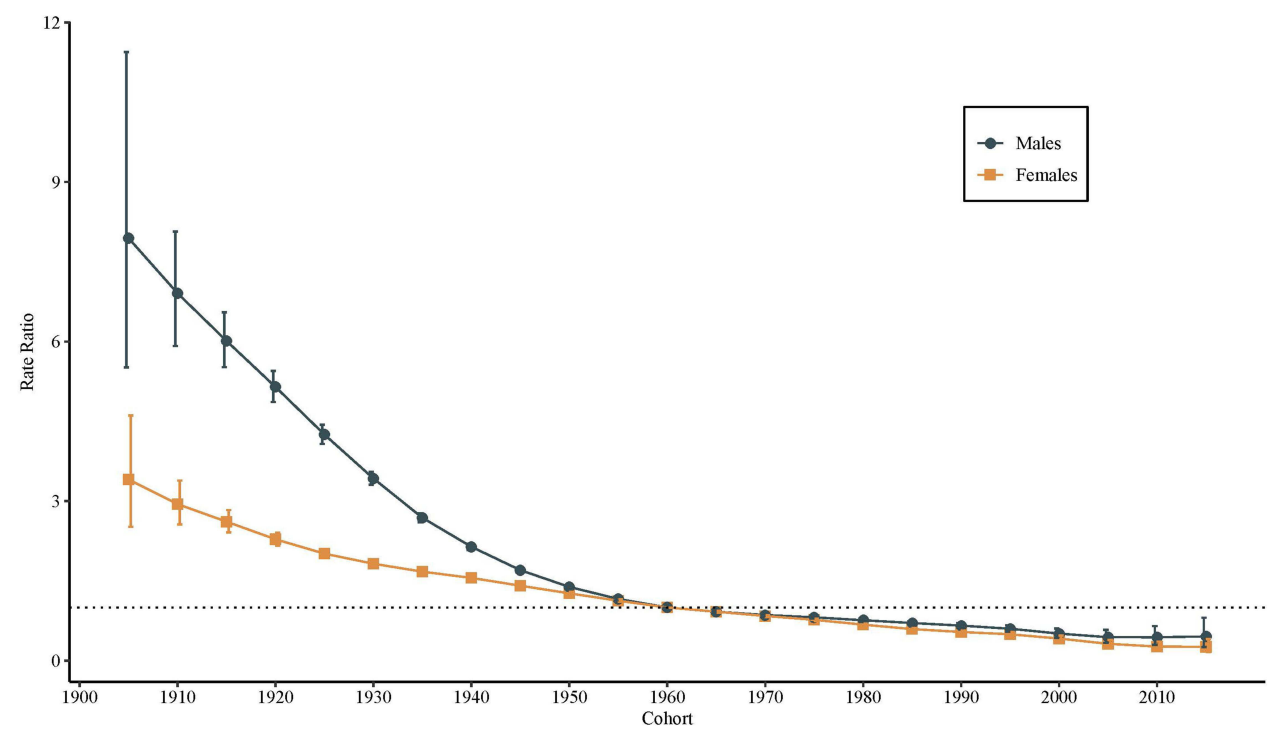

Figure 5 Cohort relative risks (RRs) of urolithiasis incidence rate by sex in China. The relative risk of each cohort compared with the reference one (cohort 1953 to 1957) adjusted for age and nonlinear period effects and the corresponding $95 \%$ confidence intervals.

acid and uronic acid are combined with calcium chelating agents to reduce calcium absorption, inhibit the aggregation of calcium oxalate crystals and cell adhesion, thereby inhibiting the formation of stones. ${ }^{21,22}$ Along with economic development, regular medical evaluations for preventative advice or appropriately timed treatment are more acceptable to the Chinese population, which would help make prevention strategies for high-risk populations and help reduce the incidence rate.

In this study, the gap between ASIR and CIR may be related to the changes in China's population structure in the past 30 years. Due to the low fertility rate caused by family planning, and the increase in life expectancy, China's population structure changed drastically, from the ideal pyramid shape in 1950 to the enlarged middle and top pyramid at the beginning of this century. ${ }^{23,24}$ And middle-aged people are the high-risk group of urolithiasis, ${ }^{6}$ which leads to an increase in the overall number of patients, and contributes to higher CIR. Considering that the age structure of China's population will continue to develop towards an aging population, ${ }^{23,25}$ urolithiasis may bring huge burdens and challenges to Chinese society. 
In this study, we found a certain sex difference between the incidence of urolithiasis. This result was consistent with the previous studies. ${ }^{6}$ It may be associated with different dietary habits. Men are more likely to consume more meat and have excessive alcohol than women. ${ }^{6}$ Interestingly, this sex difference was shrink in recent years. The same phenomenon has been found in Japan. ${ }^{26}$ This may relate to narrowed diet, lifestyle, and occupations between men and women with the development of the economy and improved living standards. ${ }^{6}$

Age is an important risk factor affecting the occurrence of urolithiasis. ${ }^{6}$ Our research results showed that in the same birth cohort and after adjusting the period deviation, the risk of urolithiasis in both males and females increased with age, and then decreased after reaching a peak. This pattern are similar to that in the United States, ${ }^{2} \operatorname{Japan}^{27}$ and other Asian countries. ${ }^{6}$ The higher risk of urolithiasis in middle-aged people may be due to their heavy workload, more physical energy consumption, and less body fluid intake, which may related to dehydration. ${ }^{6}$ Middle-aged people's unhealthy lifestyle (irregular diet or staying up late) and heavy occupational stress are also related to urolithiasis. ${ }^{26}$ This study shows that the risk of urolithiasis in men in most age groups is higher than in women, and this phenomenon has also been reported in many other countries. ${ }^{6,27,28}$ This phenomenon may be due to the fact that men prefer to drink alcohol and coffee, and consume more meat than women. ${ }^{6}$

Although under certain restrictions, period effect and cohort effect can be estimated as period RR and cohort RR, respectively. In fact, it is not easy to interpret them separately in the real world. Because when the period effect affects all age groups at the same time, it often affects a certain age group more or less, which leads to a cohort effect to a certain extent. ${ }^{12}$ Therefore, we systematically analyzed the reasons for the period effect and cohort effect trends during this period. Period effect reflects the immediate impact of social factors on the incidence of diseases. ${ }^{29}$ In this study, the period effect decreased after 2005 compared with 2000 to 2004, which may relate to one or more achievements in the field of public health in China. In 1989, China launched the "Chinese Dietary Guideline", and launched the revision version in 1997, 2007, and 2016, separately. On the basis of guideline of frequently eat milk, beans or their products in the 1997 edition, the 2007 edition added the guideline of daily drinking water and salt restrictions, and emphasized strengthening physical activity to improve people's nutritional status and reduce or prevent the occurrence of chronic diseases. Studies have shown that diet and lifestyle are closely related to the occurrence of urolithiasis. ${ }^{30}$ The implementation of these measures had improved the nutritional status of Chinese residents in a certain extent, and partially explained the decline in the risk of urolithiasis in China in recent years. The decrease in cohort effect may related to the rapid economic development of China in recent decades, the changes in residents' lifestyles, the improvement of sanitary conditions, and the formation of healthy living habits.

Some limitations of the present study should be noted. First, although there are a lots of methods in GBD 2019 to enhance the data quality, the completeness and accuracy of urolithiasis incidence data in this study may have some bias. But it can be said that the bias in this study has been reduced to a certain extent compared with the study using the original data without those correction and adjustment steps. ${ }^{12}$ Second, the data of urolithiasis in this study were estimates at the national macro level, and the macro-level explanations may not be applicable to individuals. Therefore, related hypotheses raised in this study still need further confirmation in future individualbased studies.

\section{Conclusion}

In summary, although the ASIR of urolithiasis in both Chinese men and women has decreased from 1990 to 2019. And the estimated period and cohort effects were found in similar downward patterns for both sexes. However, possibly due to changes in the demographic structure, the CIR of urolithiasis among Chinese women in the past 30 years has increased. Considering the aging of population in China, which leads to an increase in the overall number of patients, and contributes to higher CIR, urolithiasis may have huge impact on China health systems, more efforts are needed for Chinese women's urolithiasis problems.

\section{Ethics Statement}

The GBD 2019 data are freely available to the world's researchers and policymakers (http://ghdx.healthdata.org/ gbd-2019). This is a database study that reuses data from GBD 2019 to solve a research problem. It is observational and does not require ethical approval and consent.

\section{Acknowledgments}

We are grateful to individuals who participated in the study.

\section{Disclosure}

The authors report no conflicts of interest in this work. 


\section{References}

1. Chung MJ. Urolithiasis and nephrolithiasis. JAAPA. 2017;30 (9):49-50. doi:10.1097/01.JAA.0000522145.52305.aa

2. Alexander RT, Hemmelgarn BR, Wiebe N, et al. Kidney stones and kidney function loss: a cohort study. BMJ. 2012;345:e5287. doi:10.1136/bmj.e5287

3. Hsiao CY, Chen TH, Lee YC, et al. Urolithiasis is a risk factor for uroseptic shock and acute kidney injury in patients with urinary tract infection. Front Med. 2019;6:288. doi:10.3389/fmed.2019.00288

4. Saigal CS, Joyce G, Timilsina AR, Urologic Diseases in America P. Direct and indirect costs of nephrolithiasis in an employed population: opportunity for disease management? Kidney Int. 2005;68 (4):1808-1814. doi:10.1111/j.1523-1755.2005.00599.x

5. Raheem OA, Khandwala YS, Sur RL, Ghani KR, Denstedt JD. Burden of urolithiasis: trends in prevalence, treatments, and costs. Eur Urol Focus. 2017;3(1):18-26. doi:10.1016/j.euf.2017.04.001

6. Liu Y, Chen Y, Liao B, et al. Epidemiology of urolithiasis in Asia. Asian J Urol. 2018;5(4):205-214. doi:10.1016/j.ajur.2018.08.007

7. Zeng G, Mai Z, Xia S, et al. Prevalence of kidney stones in China: an ultrasonography based cross-sectional study. BJU Int. 2017;120 (1):109-116. doi:10.1111/bju.13828

8. Zeng Q, He Y. Age-specific prevalence of kidney stones in Chinese urban inhabitants. Urolithiasis. 2013;41(1):91-93. doi:10.1007/ s00240-012-0520-0

9. Vos T, Lim SS, Abbafati C, et al. Global burden of 369 diseases and injuries in 204 countries and territories, 1990-2019: a systematic analysis for the Global Burden of Disease Study 2019. Lancet 2020;396(10258):1204-1222.

10. Liu S, Wu X, Lopez $\mathrm{AD}$, et al. An integrated national mortality surveillance system for death registration and mortality surveillance, China. Bull World Health Organ. 2016;94(1):46-57. doi:10.2471/ BLT.15.153148

11. Cui F, Shen L, Li L, et al. Prevention of chronic hepatitis B after 3 decades of escalating vaccination policy, China. Emerg Infect Dis. 2017;23(5):765-772. doi:10.3201/eid2305.161477

12. Wang Z, Hu S, Sang S, Luo L, Yu C. Age-period-cohort analysis of stroke mortality in China: data from the Global Burden of Disease Study 2013. Stroke. 2017;48(2):271-275. doi:10.1161/ STROKEAHA.116.015031

13. Yuan S, Shi Y, Li M, Hu X, Bai R. Trends in incidence of urinary tract infection in Mainland China from 1990 to 2019. Int J Gen Med. 2021;14:1413-1420. doi:10.2147/IJGM.S305358

14. Rosenberg PS, Check DP, Anderson WF. A web tool for age-periodcohort analysis of cancer incidence and mortality rates. Cancer Epidemiol Biomarkers Prev. 2014;23(11):2296-2302. doi:10.1158/ 1055-9965.EPI-14-0300

15. Cao J, Eshak ES, Liu K, Gero K, Liu Z, Yu C. Age-period-cohort analysis of stroke mortality attributable to high sodium intake in China and Japan. Stroke. 2019;50(7):1648-1654. doi:10.1161/ STROKEAHA.118.024617

International Journal of General Medicine

\section{Publish your work in this journal}

The International Journal of General Medicine is an international, peer-reviewed open-access journal that focuses on general and internal medicine, pathogenesis, epidemiology, diagnosis, monitoring and treatment protocols. The journal is characterized by the rapid reporting of reviews, original research and clinical studies

Submit your manuscript here: https://www.dovepress.com/international-journal-of-general-medicine-journal
16. Huang WY, Chen YF, Carter S, Chang HC, Lan CF, Huang KH. Epidemiology of upper urinary tract stone disease in a Taiwanese population: a nationwide, population based study. $J$ Urol. 2013;189 (6):2158-2163. doi:10.1016/j.juro.2012.12.105

17. Lee YH, Huang WC, Tsai JY, et al. Epidemiological studies on the prevalence of upper urinary calculi in Taiwan. Urol Int. 2002;68 (3):172-177. doi:10.1159/000048445

18. Xiao Y, Su C, Ouyang Y, Zhang B. Trends of vegetables and fruits consumption among Chinese adults aged 18 to 44 years old from 1991 to 2011. Chin J Epidemiol. 2015;36(3):232-236.

19. Wang $\mathrm{ZH}$, Zhang B, Wang HJ, et al. Trend in vegetable and fruit intake among Chinese children and adolescents aged 6 to 17 years from 1991 to 2009 and related socio-demographic factors. Chin J Epidemiol. 2013;34(9):863-868.

20. Taylor EN, Fung TT, Curhan GC. DASH-style diet associates with reduced risk for kidney stones. J Am Soc Nephrol. 2009;20 (10):2253-2259. doi:10.1681/ASN.2009030276

21. Ngo TC, Assimos DG. Uric acid nephrolithiasis: recent progress and future directions. Rev Urol. 2007;9(1):17-27.

22. Tsujihata M, Miyake O, Yoshimura K, Kakimoto KI, Takahara S, Okuyama A. Fibronectin as a potent inhibitor of calcium oxalate urolithiasis. J Urol. 2000;164(5):1718-1723. doi:10.1016/S00225347(05)67095-4

23. Zhang NJ, Guo M, Zheng X. China: awakening giant developing solutions to population aging. Gerontologist. 2012;52(5):589-596. doi:10.1093/geront/gns105

24. Howe N, Jackson R. Global aging and the crisis of the 2020s. Curr Hist. 2011;110(732):20-25. doi:10.1525/curh.2011.110.732.20

25. Bai R, Wu W, Dong W, Liu J, Yang L, Lyu J. Forecasting the populations of overweight and obese Chinese adults. Diabetes Metab Syndr Obes. 2020;13:4849-4857. doi:10.2147/DMSO. S274110

26. Iguchi M, Umekawa T, Katoh Y, Kohri K, Kurita T. Prevalence of urolithiasis in Kaizuka City, Japan--an epidemiologic study of urinary stones. Int J Urol. 1996;3(3):175-179. doi:10.1111/j.1442-2042.1996. tb00511.x

27. Yasui T, Iguchi M, Suzuki S, Kohri K. Prevalence and epidemiological characteristics of urolithiasis in Japan: national trends between 1965 and 2005. Urology. 2008;71(2):209-213. doi:10.1016/j. urology.2007.09.034

28. Hussein NS, Sadiq SM, Kamaliah MD, Norakmal AW, Gohar MN. Twenty-four-hour urine constituents in stone formers: a study from the northeast part of Peninsular Malaysia. Saudi J Kidney Dis Transpl. 2013;24(3):630-637. doi:10.4103/1319-2442.111090

29. Yang J, Zhang Y, Luo L, Meng R, Yu C. Global mortality burden of cirrhosis and liver cancer attributable to injection drug use, 19902016: an age-period-cohort and spatial autocorrelation analysis. Int $J$ Environ Res Public Health. 2018;15:1.

30. Eisner BH, Goldfarb DS. A nomogram for the prediction of kidney stone recurrence. $J$ Am Soc Nephrol. 2014;25(12):2685-2687. doi:10.1681/ASN.2014060631 across all disease areas. The manuscript management system is completely online and includes a very quick and fair peer-review system, which is all easy to use. Visit http://www.dovepress.com/ testimonials.php to read real quotes from published authors. 